\title{
Diversity of floral regulatory genes of japonica rice cultivated at northern latitudes
}

\author{
Laura Naranjo, Manuel Talón and Concha Domingo*
}

\begin{abstract}
Background: Rice is considered a short day plant. Originally from tropical regions rice has been progressively adapted to temperate climates and long day conditions in part by modulating its sensitivity to day length. Heading date $3 a$ (Hd3a) and RICE FLOWERING LOCUS T 1 (RFT1) that code for florigens, are known as major regulatory genes of floral transition in rice. Both Hd3a and RFT1 are regulated by Early heading date 1 (Ehd1) and Days to heading on chromosome 2 (DTH2) while Heading date 1 (Hd1) also governs Hd3a expression. To investigate the mechanism of rice adaptation to temperate climates we have analyzed the natural variation of these five genes in a collection of japonica rice representing the genetic diversity of long day cultivated rice.

Results: We have investigated polymorphisms of Hd3a, RFT1, Ehd1, Hd1 and DTH2 in a collection of 57 japonica varieties. Hd3a and RFT1 were highly conserved, displaying one major allele. Expression analysis suggested that RFT1 rather than $\mathrm{Hd} 3$ a could be the pivotal gene controlling flowering under long day conditions. While few alleles were found in the Ehd1 promoter and DTH2 coding region, a high degree of variation in Hd1, including non-functional alleles, was observed. Correlation analysis between gene expression levels and flowering periods suggested the occurrence of other factors, additionally to Ehd1, affecting RFT1 regulation in long day adapted cultivars.

Conclusions: During domestication, rice expansion was accompanied by changes in the regulatory mechanism of flowering. The existence of non-functional Hdl alleles and the lack of correlation of their presence with flowering times in plants grown under long day conditions, indicate a minor role of this branch in this process and the existence of an alternative regulatory pathway in northern latitudes. Expression analysis data and a high degree of conservation of RFT1 suggested that this gene could be the main factor regulating flowering among japonica cultivars adapted to northern areas. In the absence of inhibition exerted by Hd1 through repression of Hd3a expression, the role of Ehd1 as a regulator of RFT1 and Hd3a appears to be reinforced. Data also indicated the occurrence of additional regulatory factors controlling flowering.
\end{abstract}

Keywords: Flowering, Short day, Rice, Polymorphism, Natural variation

\section{Background}

Modern rice (Oryza sativa L.) originated in southern China and, accompanying human migration, was expanded to a wide range of geographical regions [1] diverging into indica, japonica and aus types. These genetic groups are characterized by specific climate adaptation, according to the agroecological conditions where they were cultivated. The indica genotypes are grown in tropical climates whereas japonica genotypes, as they suffered a wider expansion, can be found either in tropical or temperate climates. The adaptation process to new

\footnotetext{
*Correspondence: domingo_concar@gva.es

Instituto Valenciano de Investigaciones Agrarias, Carretera Moncada-Naquera Km 4,5, Moncada 46113, Spain
}

(c) 2014 Naranjo et al.; licensee BioMed Central Ltd. This is an open access article distributed under the terms of the Creative Commons Attribution License (http://creativecommons.org/licenses/by/2.0), which permits unrestricted use, distribution, and reproduction in any medium, provided the original work is properly cited. conferred ad and transmitted through generations. Reaching northern limits of its natural cultivation area, japonica rice was progressively adapting to short growing seasons. In these new areas, flowering under long days (LD) allowed cultivation in summer thus avoiding cold winter temperatures. Genes involved in flowering regulation should show allelic differentiation across environmental gradients and the frequency in the allele pool present in different geographical regions may reflect the mechanism of adaptation of plants to new conditions of day length.

The regulation of flowering is a fine tuned mechanism that changed as part of the adaptation process to new 
climate conditions. Two independent photoperiod pathways, involving the floral regulators Heading date 1 (Hd1) and Early heading date 1 (Ehd1), have been implicated in flowering date. These pathways converge through modulation of the expression of Heading date $3 a(H d 3 a)$ or RICE FLOWERING LOCUS T1 (RFT1), the genes that encode florigen, a mobile signal that moves from the leaves to the apical meristem to switch on the flowering process [2,3]. $H d 1$ is predominantly regulated by the circadian clock through OsGIGANTEA $(O s G I)$, a key gene that positively controls $H d 1$ expression [4]. Hd1 is a zinc finger type transcriptional activator with a conserved CCT-domain that acts as a crucial nuclear localization signal for the correct protein function [5]. It has been proposed that the presence of lightactive phytochromes could modify $\mathrm{Hd} 1$ activity to inhibit $H d 3 a$ transcription [6]. Under SD conditions, Hd1 activates $H d 3 a$ transcription thereby promoting flowering, whereas under non-inductive LD conditions Hd1 acts as a repressor [6,7]. This claim is supported by the fact that loss-of-function $H d 1$ mutants display early and late heading dates when grown under LD and SD conditions, respectively [8]. Hd1 protein can also be modified in the presence of Heading date 6 protein (Hd6) suppressing $H d 3 a$ expression, but only when $\mathrm{Hd} 1$ is functional [9]. Hd6 shows casein kinase activity, but there is evidence that Hd6 does not phosphorylate directly Hd1 suggesting the action of an additional factor. Ehd1 is unique to the flowering pathway in rice and codes for a $B$-type response regulator that, in an $H d 1$-independent manner, induces flowering under both SD and LD conditions [10]. Expression analysis using transgenic plants over-expressing Ehd1 indicate that Ehd1 activates transcription of $H d 3 a$ in rice, as well as other additional rice orthologs of Arabidopsis FT genes [10]. Ehd1 is thought to be a signal integrator of floral transition as multiple regulatory pathways convey through Ehd1 to regulate $H d 3 a$ or RFT1 expression. In part, OsGI, the circadian clock mediator, regulates Ehd1 expression under SD conditions via OsMADS51 [11]. OsMAD50, another MADS box gene, is a positive regulator of Ehd1 expression, and it works either parallel with or downstream of OsGI $[12,13]$. Ghd8/DTH8, was identified as a major QTL with pleiotropic effects on grain yield, heading date and plant height. This QTL appears to delay flowering under LD conditions, and promote it in the opposite conditions. Moreover, Ghd8/DTH8 also represses the expression of Ehd1 and $H d 3 a$ under LD conditions, but it is not clear if the QTL acts directly on $H d 3 a$ or down-regulates Ehd1 [14,15]. It is also known that Oryza sativa LEC1 and FUSCA-LIKE 1 (OsLFL1) and Ghd7 delays flowering under LD conditions, most likely repressing Ehd1 [16,17]. In a different pathway, Ehd2/OsId1 up-regulates Ehd1 expression under both SD and LD conditions [18,19]. Early heading date 4 (Ehd4) protein is localized in the nucleus and has acid-binding and transcriptional activation properties. Furthermore, Ehd4 promotes flowering increasing Ehd1 expression [20]. On the other hand, phytochromes inhibit flowering by affecting both Hd1 and Ehd1 regulation. PHOTOPERIOD SENSITIVITY5 (SE5), for example, that encodes a heme oxygenase involved in the biosynthesis of the chromophore part of phytochrome, represses Ehd1 expression and therefore acts as a floral repressor [21].

It has been shown that the expression pattern of $\mathrm{Hd} 1$ is clearly distinct from that of Ehd1. Hd1, which functions as a circadian clock mediator, shows similar patterns of expression under both SD and LD conditions whereas Ehd1 is mainly induced under SD conditions [10]. These observations imply that, in rice, $H d 1$ and Ehd1 are antagonistic under LD, but are synergistic under SD. Hd1, depending upon phytochrome abundance, promotes flowering under SD and inhibits flowering under LD, whereas Ehd 1 promotes flowering under both conditions $[10,22]$. It has been suggested that the equilibrium between the influence of both pathways that converge on $H d 1$ and Ehd 1 results in $H d 3 a$ modulation and, consequently, in the regulation of floral transition under SD conditions [22].

$H d 3 a$ is a member of the FT-like family and shares 91\% of identity with RFT1, the closest homologue. Although there is evidence that FT-L1/FTL is possibly involved in flowering [6,23], $H d 3 a$ and RFT1 are considered to be the major contributors of the FT-like members to floral promotion since suppression of both $H d 3 a$ and RFT1 expression results in no flowering [23]. Although it has been demonstrated that RFT1 can certainly activate flowering in the absence of $H d 3 a$ expression under SD conditions, a secondary role however has been given to RFT1 in floral promotion under SD conditions [23]. On the other side, RFT1 seems to be a major floral activator under LD conditions as evidenced by RNAi studies where expression of either $H d 3 a, R F T 1$ or both was suppressed [3]. In addition, this is supported by the fact that SDG724, a rice histone methyltransferase gene, that is required for flowering under LD conditions modulates positively EHd1 and RFT1 expression, but not $H d 3 a$ [24]. Despite all these major factors involved in the flowering regulation, some other factors affecting florigen regulation should be considered. Recently, Days to heading on chromosome 2 (DTH2), a minor-effect quantitative trait locus that promotes heading under LD conditions has been cloned. DTH2 encodes a CONSTANT-like protein that promotes flowering by inducing $\mathrm{Hd} 3 \mathrm{a}$ and RFT1 independently of $H d 1$ and Ehd1 [25].

Diversification of flowering time was one of the major reasons for rice expansion. Selection of favorable alleles during the domestication and breeding process lead to the generation of new varieties adapted to different 
climate conditions. In this regard, the identification and characterization of allele diversity in rice populations, offers information of relevance on the mechanism of adaptation of these plants. Studies on variation of flowering regulatory genes have previously been performed on the three pivotal genetic factors contributing to flowering time in rice under SD: the promoter type of $H d 3 a$, the functionality of $H d 1$ and the expression level of Ehd1 $[26,27]$. These studies indicated that changes in flowering time are probably not dependent on the intrinsic features of the florigens, but rather on their regulation. Hd3a protein function has been shown to be highly conserved among rice cultivars since no functional changes were found in $\mathrm{Hd} 3 \mathrm{a}$ in either cultivated or ancestral rice. Additionally, $\mathrm{Hd} 3 \mathrm{a}$ expression level showed a strong correlation with flowering time under SD conditions $[27,28]$, suggesting that differences in the $H d 3 a$ promoter type, rather than protein variation, directly affects the variation in $H d 3 a$ expression levels. Similarly to $H d 3 a$, Ehd 1 also encodes a highly conserved protein in cultivated rice. Although full evidence is still lacking, there are indications that variations in genes functioning upstream of Ehd1 in the regulatory pathway, such as Ghd7 or MADS50, could potentially provide variations in Ehd1 expression [27,29]. In contrast, a high degree of polymorphism has been found in $H d 1$, that possesses several non-functional alleles. Loss-of function alleles of Hd1 are common in cultivated rice and it has been suggested that they cause flowering time diversity [28]. Analysis of variation of $H d 1$ in modern and ancient cultivars indicated that multiple introgression events in certain alleles of $H d 1$, including non-functional alleles, played a role in adaptation to specific areas at the beginning of domestication [30].

Despite all the information available, most of variation analyses of flowering regulatory genes have been performed with indica rice while little is known on the diversity in japonica varieties adapted to LD conditions. Additionally, the characterization of the allele pool could reveal information about the role of RFT1 on induction of flowering in northern latitudes. To better understand the mechanism of flowering of japonica rice, cultivated in Northern latitudes adapted to LD conditions, in this study we analyzed the allele distribution and the expression of the master genes, RFT1 and $H d 3 a$, and their immediately regulators, $H d 1$, Ehd 1 and DTH2.

\section{Results}

\section{Cycle length of japonica cultivars}

To analyze the genetic pool related to flowering control in japonica rice adapted to LD conditions, a core collection of 57 selected cultivars was used. The collection included japonica type cultivars from different geographical origins, mainly from Northern latitudes, and two indica cultivars, as control. Cultivars were representative of all genetic groups found across Northern latitudes according to recent population structure analysis [31]. Plants grown under natural LD conditions during summer were able to reach the reproductive stage and to produce panicles, except Kinmaze plants. Plants flowered ranging from 73 days to 168 days after sowing and had an average panicle emergence of 115 days after sowing (Figure 1).

\section{Expression analysis of RFT1 and $\mathrm{Hd} 3 \mathrm{a}$}

To study the role of RFT1 and $H d 3 a$ in the induction of flowering in japonica cultivars under LD conditions, we searched for possible relationships between their expression levels and flowering times by performing an expression analysis in 11 weeks old plants of our core collection. Expression levels of both RFT1 and $\mathrm{Hd} 3 a$ were variable among cultivars. mRNA levels of RFT1 were most closely correlated with flowering time, indicating that higher expression levels were associated with earlier flowering time $\left(\mathrm{n}=56, \mathrm{R}^{2}=0,6369\right) \quad$ (Figure 2A). Hd3a mRNA levels showed little correlation with flowering time of the different cultivars $\left(n=56, R^{2}=0,2294\right)$ (Figure 2B), suggesting that $H d 3 a$ expression has lower effect on flowering regulation of japonica rice cultivated under LD conditions. Therefore, RFT1 seems to contribute as the main factor in the regulation of transition from vegetative to reproductive phase under LD conditions among japonica cultivars while $H d 3 a$, also expressed, participates with a minor role. These data are consistent with previous results showing that RFT1 is a major floral activator in rice grown under LD conditions [3].

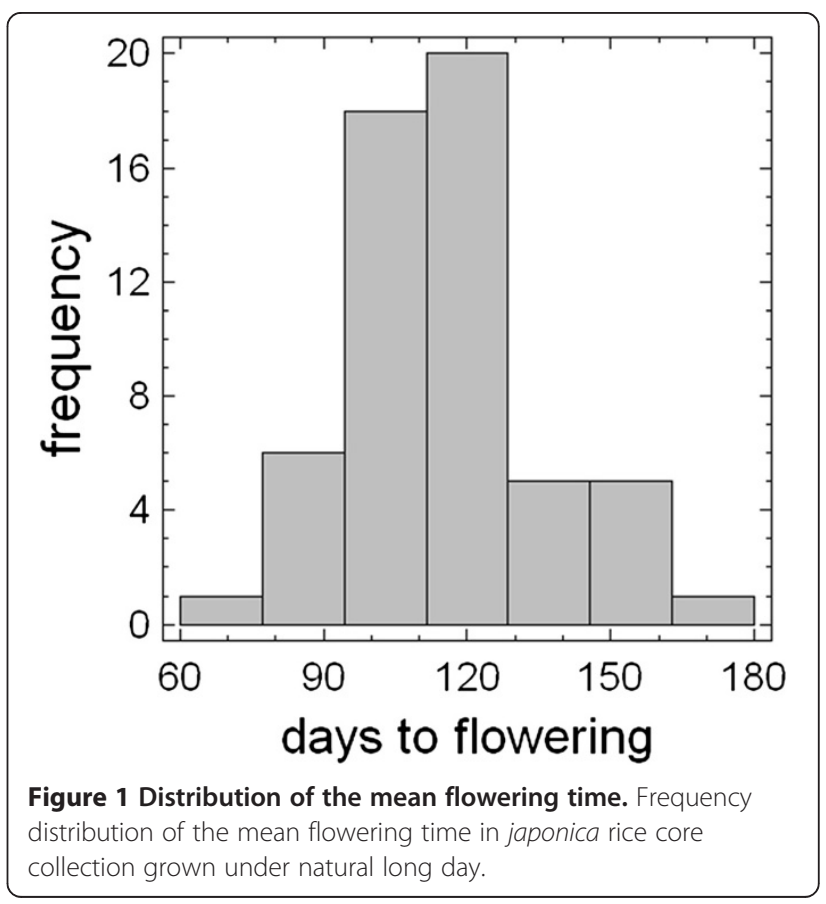




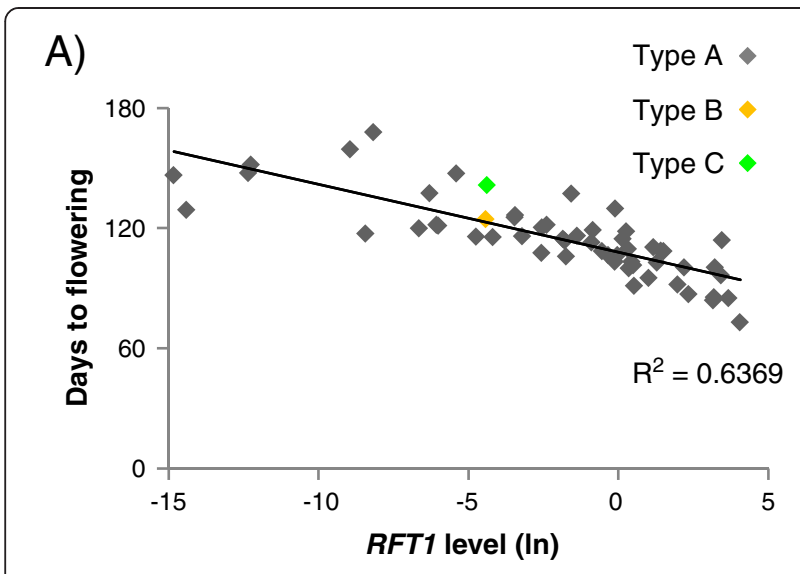

B)

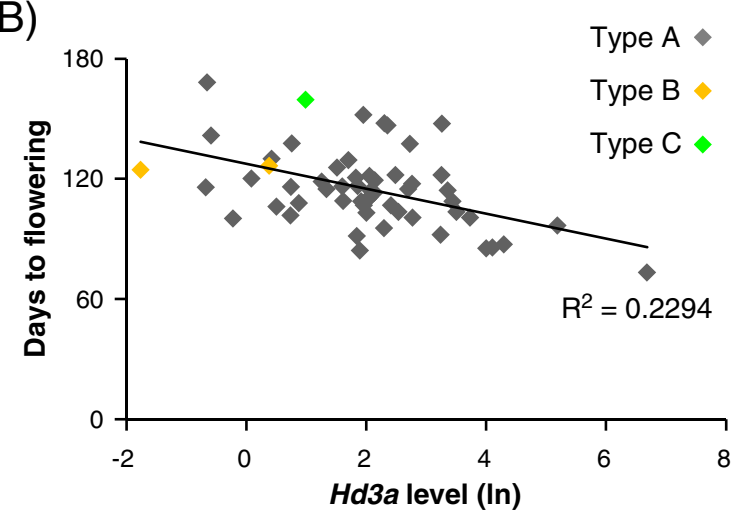

Figure 2 Correlation of flowering time with RNA levels of $H d 3 a$ or RFT1. Correlation of flowering time with RNA levels of (A) RFT1 or (B) Hd3a in leaves. RNA levels were determined by real-time RT-PCR and shown as natural logarithms. The square of Pearson's productmoment correlation coefficient is indicated $(P<0,05)$. Color dots indicate cultivars carrying different types of RFT1 or Hd3a alleles.

Nucleotide polymorphism in the promoter of RFT1 and $\mathrm{Hd} 3 a$ We investigated the occurrence of nucleotide polymorphisms in the RFT1 promoter region including the 5'untraslated region (UTR) and found a high degree of conservation (Figure 3A). One major allele was shared by all cultivars in the core collection, except by two of them. One of the alleles differed only in a single nucleotide deletion. Kasalath, included in the core collection as a control, contained a third allele showing differences in the promoter region, including the UTR, consisting in single nucleotide substitutions, a deletion of $11 \mathrm{bp}$ and a insertion of $22 \mathrm{bp}$. Despite variations in RFT1 promoter in Kasalath, the expression levels of RFT1 followed the tendency observed in other cultivars and did not appear to affect expression (Figure 2A). As little is known about variability of the coding region of RFT1, we further investigated this region. We detected the presence of a unique allele among all japonica cultivars. Again, Kasalath showed differences that, in this case, implicated two amino acid changes in the encoded protein (Figure 3B).
Previous studies found little variability in the protein encoded by $H d 3 a$ and variations found in the coding region did not affect the functional domains of the protein $[4,27]$. To investigate the diversity in the promoter region of $H d 3 a$ in japonica cultivars, we analyzed in the core collection over $2-\mathrm{kb}$ region of $\mathrm{Hd} 3 \mathrm{a}$ including the promoter and the 5_UTR region by EcoTilling. Nucleotide polymorphism analysis revealed that $\mathrm{Hd} 3 a$ was also highly conserved among japonica cultivars (Figure 3C). All cultivars included in this study shared the same $H d 3 a$ promoter sequence with the exception of three that contained two different alleles. As RFT1allele, Kasalath contained a different $H d 3 a$ allele to the rest of the core collection (Table 1). Variations identified in the $\mathrm{Hd} 3 \mathrm{a}$ promoter consisted of single nucleotide substitutions, small deletions and a $12 \mathrm{bp}$ insertion. No major alterations in potential regulatory sequences of the $H d 3 a$ promoter were identified. Within the analyzed promoter fragment, no changes affected the CCAAT box or the three binding sites recognizing the GARP DNA-binding domain of Ehd 1 that potentially can bind the $H d 3 a$ promoter via the ARR1 binding element [10]. The variability found in $H d 3 a$ promoter among the analyzed cultivars was lower than in previous studies using indica cultivars [27].

\section{Nucleotide polymorphism in Ehd1, Hd1 and DTH2}

In a search for nucleotide polymorphisms that may cause differences in Ehd1 expression, we analyzed $1 \mathrm{~kb}$ of Ehd1 promoter region by EcoTilling. We identified 3 types of sequences among japonica cultivars (Figure 4A); although only two of them were present predominantly. Type A was shared by 36 cultivars while type B was found in 17 cultivars, and both types were widely distributed along all continents (Table 1 ). Type $\mathrm{C}$ was exclusively present in four cultivars; two of them were indica type and a third had indica ancestors suggesting that allele $C$ is characteristic of indica rice. Differences between the three types of alleles consisted of single nucleotide substitutions or insertions. Expression analyses showed that Ehd1 mRNA levels varied among cultivars and showed moderate correlation with the length of cycle ( $\mathrm{n}=56, R^{2}=0,273$ ) (Figure 4B), indicating that other factors than Ehd 1 were regulating flowering promotion under LD conditions. Additionally, despite the extent of variation, allele type A tended to show higher expression levels than allele $\mathrm{B}$ or $\mathrm{C}$ as shown in Figure $4 B$ and $C$, indicating the possibility of association between promoter type and expression level.

To look for changes in Hd1 that may alter the capacity of function of the protein, we analyzed polymorphisms in $H d 1$ coding sequence among the core collection and we found a high degree of variation, leading to the identification of 14 different alleles (Figure 5A). Almost half of the analyzed cultivars, 23 out of 57 cultivars, contained $H d 1$ variants that are likely to contain non- 


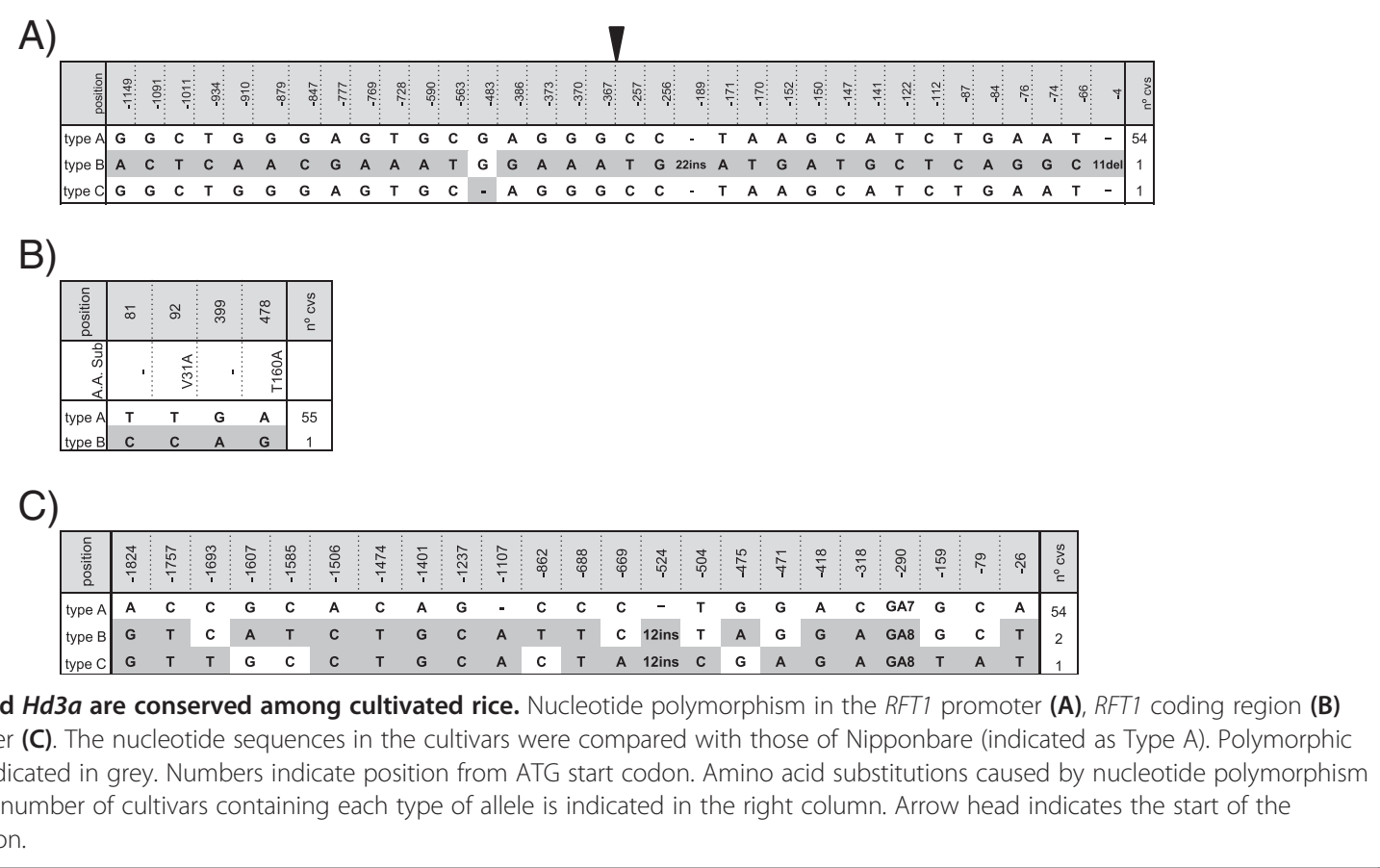

functional alleles of $H d 1$. Most of the loss of function was due to deletions that caused frame-shift mutations or changes in one nucleotide that created premature stop codons. Additionally, a three base pair deletion localized within the CCT-domain of the protein that acts as a nuclear localization signal was present in ten cultivars. In these varieties, the correct function of $\mathrm{Hd} 1$ could be affected [5]. Other variants affecting other regions of the protein could also cause changes in the protein activity. This is the case of a deletion of 9 amino acids at the $\mathrm{N}$-terminus of the zinc finger domain in allele 12, that may cause deregulation in Hd1 binding capacity or alterations in the protein recognition capacity. C81Y substitution, in alleles 13 and 14, also affects the zinc finger domain. On the other hand, R83Q and H106Y changes have been reported previously, but no effect on the Hd1 function was detected [27]. Hd1 alleles were distributed randomly throughout all geographical regions (Table 1), regardless of whether they carry functional or nonfunctional $H d 1$ alleles.

To determine whether variations in the flowering time of japonica cultivars grown under LD conditions were correlated with the allelic diversity of $H d 1$, we grouped all the cultivars into those with functional and nonfunctional $H d 1$ alleles and compared their RFT1 and $H d 3 a$ mRNA levels with flowering times (Figure $5 \mathrm{~B}$ ). No correlation could be found ( $\mathrm{n}=56, \mathrm{P}>0,05)$ neither between RFT1 or $H d 3 a$ mRNA levels in cultivars, carrying functional or non-functional $H d 1$ alleles, and flowering time. High or low levels or mRNA were found in cultivars independently of the functionality of the displayed
$H d 1$ allele. These results suggested a minor influence of $H d 1$ on RFT1 and Hd3a regulation in flowering induction among the analyzed cultivars grown under LD conditions. This irrelevant role of $H d 1$ would be in accordance with the presence of a high number of $H d 1$ variants compared to RFT1, Hd3a and Ehd1, the other genes implicated in flowering pathway regulation. This is reinforced by the fact that there was no significant difference in flowering time between cultivars displaying functional or non-functional $H d 1$ alleles, (flowering on average $110,4 \pm 17,7$ or $122,1 \pm 20,2$ days after showing, respectively).

To investigate diversity in the DTH2 that may cause differences in flowering time among the core collection, we examined DTH2 coding region and we found variations in four nucleotides. Type A was present in $47 \mathrm{cul}-$ tivars (Figure 6A). Type B1 and B2 differed in a synonymous SNP at position 1.645 and, thus, they were grouped as type $\mathrm{B}$, and was found in 8 cultivars. Type $\mathrm{C}$ was present only in one cultivar and contained a SNP that implicated an amino acid substitution (R9G) that affected the zinc finger domain at the $\mathrm{N}$-terminal region of the protein [25]. We analyzed flowering time of $j a-$ ponica cultivars grown under LD conditions carrying DTH2 type A or type B alleles and found no significant differences $(P>0,1)$ in flowering time between both groups of cultivars (Figure 6B).

\section{Discussion}

Rice is considered a short day plant, as its optimal flowering conditions are given under short day photoperiod. 
Table 1 Flowering times and allele types of genes analyzed in the cultivars of the core collection

\begin{tabular}{|c|c|c|c|c|c|c|c|c|c|}
\hline & Variety & Country & & $\begin{array}{l}\text { Days to } \\
\text { flowering }\end{array}$ & $\begin{array}{c}\text { Hd3a } \\
\text { promoter } \\
\text { sequence }\end{array}$ & $\begin{array}{l}\text { RFT1 promoter } \\
\text { and coding } \\
\text { sequence }\end{array}$ & $\begin{array}{c}H d 1 \\
\text { coding } \\
\text { sequence }\end{array}$ & $\begin{array}{c}\text { Ehd1 } \\
\text { promoter } \\
\text { sequence }\end{array}$ & $\begin{array}{c}D T H 2 \\
\text { coding } \\
\text { sequence }\end{array}$ \\
\hline 1 & Agami & Egypt & Africa & $168,0 \pm 1,5$ & $\mathrm{a}$ & $\mathrm{a}$ & 9 & a & $a$ \\
\hline 2 & Moroberekan & Guinea & Africa & $147,5 \pm 5,2$ & a & a & 9 & $b$ & a \\
\hline 3 & GZ178 & Egypt & Africa & $116,0 \pm 1,4$ & a & a & 2 & c & b2 \\
\hline 4 & Hwayoung & Korea & Asia & $107,7 \pm 2,3$ & a & a & 1 & a & a \\
\hline 5 & Koshihikari & Japan & Asia & $106,0 \pm 6,1$ & a & a & 1 & a & a \\
\hline 6 & Nipponbare & Japan & Asia & $147,3 \pm 13,7$ & a & a & 1 & a & a \\
\hline 7 & Akihikari & Korea & Asia & $113,8 \pm 1,9$ & a & a & 3 & a & a \\
\hline 8 & Sasanishiki & Japan & Asia & $121,3 \pm 2,9$ & a & a & 3 & a & a \\
\hline 9 & Daegudo & Korea & Asia & $91,3 \pm 0,5$ & a & a & 4 & a & a \\
\hline 10 & Dongjin & Korea & Asia & $125,3 \pm 14,6$ & a & a & 4 & a & a \\
\hline 11 & Kinmaze & Japan & Asia & $>190$ & a & a & 4 & a & a \\
\hline 12 & Tainung 67 & Taiwan & Asia & $137,3 \pm 6,2$ & a & a & 4 & a & b1 \\
\hline 13 & Zonghua 15 & China & Asia & $151,8 \pm 4,6$ & a & a & 4 & a & a \\
\hline 14 & Taichung 65 & Taiwan & Asia & $159,5 \pm 0,6$ & c & a & 5 & a & $d$ \\
\hline 15 & Guweoldo & Korea & Asia & $137,5 \pm 3,7$ & a & a & 6 & a & a \\
\hline 16 & LTH & China & Asia & $101,5 \pm 2,1$ & a & a & 11 & $\mathrm{a}$ & b1 \\
\hline 17 & Azucena & Philippines & Asia & $141,5 \pm 1,9$ & a & c & 9 & b & a \\
\hline 18 & Kasalath & India & Asia & $124,5 \pm 4,1$ & $b$ & $b$ & 8 & c & b2 \\
\hline 19 & Norin 8 & Japan & Asia & $126,5 \pm 1,0$ & b & a & 8 & c & b2 \\
\hline 20 & IR64 & Philippines & Asia & $129,3 \pm 0,5$ & a & a & 9 & c & b2 \\
\hline 21 & Harra & Australia & Australia & $115,5 \pm 6,1$ & a & a & 4 & $b$ & a \\
\hline 22 & Guadiamar & Spain & Europe & $102,8 \pm 1,5$ & a & a & 3 & a & a \\
\hline 23 & Bahia & Spain & Europe & $121,8 \pm 11,3$ & a & $a$ & 4 & a & a \\
\hline 24 & Bendret & Spain & Europe & $119,0 \pm 2,9$ & a & a & 4 & a & a \\
\hline 25 & Benisants & Spain & Europe & $113,0 \pm 2,3$ & a & a & 4 & $\mathrm{a}$ & a \\
\hline 26 & Gavina & Spain & Europe & $103,3 \pm 8,5$ & a & a & 4 & a & a \\
\hline 27 & Ullal & Spain & Europe & $114,8 \pm 1,0$ & a & a & 4 & a & a \\
\hline 28 & crlb1 & Italy & Europe & $85,0 \pm 2,0$ & a & a & 9 & a & a \\
\hline 29 & Gladio & Italy & Europe & $100,5 \pm 2,4$ & a & a & 9 & a & a \\
\hline 30 & Ripallo & Spain & Europe & $103,3 \pm 1,9$ & a & a & 9 & a & a \\
\hline 31 & Carnice Precoce & Italy & Europe & $100,5 \pm 1,9$ & a & a & 10 & a & a \\
\hline 32 & Romolo & Italy & Europe & $106,5 \pm 3,0$ & a & a & 10 & a & a \\
\hline 33 & Gigante Vercelli & Italy & Europe & $87,0 \pm 2,0$ & a & a & 11 & a & a \\
\hline 34 & Giov. Marchetti & Italy & Europe & $84,0 \pm 0,0$ & a & a & 11 & a & a \\
\hline 35 & Loto & Italy & Europe & $85,5 \pm 2,4$ & a & a & 11 & a & a \\
\hline 36 & Bomba & Spain & Europe & $118,5 \pm 1,9$ & a & a & 12 & a & b1 \\
\hline 37 & Italica Livorno & Italy & Europe & $106,5 \pm 5,4$ & a & a & 13 & a & a \\
\hline 38 & Pegonil & Spain & Europe & $117,3 \pm 1,5$ & a & a & 13 & a & a \\
\hline 39 & Arroz da Terra & Portugal & Europe & $73,0 \pm 2,0$ & a & a & 14 & a & a \\
\hline 40 & Fonsa & Spain & Europe & $95,3 \pm 0,5$ & a & a & 4 & $b$ & a \\
\hline 41 & JSendra & Spain & Europe & $109,8 \pm 2,2$ & a & a & 4 & $b$ & a \\
\hline 42 & Leda & Spain & Europe & $129,8 \pm 6,4$ & a & a & 4 & $b$ & a \\
\hline 43 & Senia & Spain & Europe & $108,8 \pm 8,2$ & a & a & 4 & $b$ & a \\
\hline
\end{tabular}


Table 1 Flowering times and allele types of genes analyzed in the cultivars of the core collection (Continued)

\begin{tabular}{|c|c|c|c|c|c|c|c|c|c|}
\hline 44 & Sequial & Spain & Europe & $92,0 \pm 3,6$ & $a$ & $a$ & 4 & $b$ & a \\
\hline 45 & Campino & Portugal & Europe & $146,5 \pm 1,9$ & $a$ & $a$ & 9 & $b$ & a \\
\hline 46 & Cormorán & Spain & Europe & $115,8 \pm 3,3$ & $a$ & a & 9 & $b$ & a \\
\hline 47 & Gleva & Spain & Europe & $116,3 \pm 2,6$ & a & a & 9 & $b$ & $a$ \\
\hline 48 & Puntal & Spain & Europe & $120,3 \pm 3,1$ & $a$ & $a$ & 9 & $b$ & $a$ \\
\hline 49 & Cesare & Italy & Europe & $108,5 \pm 2,9$ & $a$ & $a$ & 11 & $b$ & $a$ \\
\hline 50 & Paulovski & Russia & Europe & $96,5 \pm 3,0$ & a & a & 14 & $b$ & b1 \\
\hline 51 & Calmochi-101 & USA & America & $100,0 \pm 2,9$ & a & a & 3 & a & a \\
\hline 52 & M201 & USA & America & $121,8 \pm 3,8$ & $a$ & a & 4 & a & a \\
\hline 53 & M202 / Thainnato & USA & America & $108,5 \pm 3,4$ & a & a & 4 & a & a \\
\hline 54 & L202 /Thaibonnet & USA & America & $120,0 \pm 2,6$ & a & a & 9 & a & a \\
\hline 55 & Lemont & USA & America & $114,0 \pm 5,8$ & a & $a$ & 9 & $b$ & a \\
\hline 56 & Gema & Puerto Rico & America & $114,8 \pm 2,4$ & a & $a$ & 7 & $b$ & a \\
\hline 57 & CT-18 & Colombia & America & $110,5 \pm 1,3$ & $a$ & $a$ & 11 & b & a \\
\hline
\end{tabular}

Flowering times and alleles types classification of Hd3a, RFT1, Ehd1, Hd1 and DTH2 in each cultivar of the core collection based on polymorphisms.

Rice was domesticated in two independent events in two locations in China, in a tropical region, and then it was extended to the rest of the world [1]. The spread of rice to a wide range of geographical areas encompassed adaptation to habitats with different climates that included large differences in photoperiod. The transition of rice from vegetative to reproductive stage under SD conditions is dictated by $H d 3 a$, the master gene that codes for one of the mobile signal of flowering [2]. Hd1 and Ehd 1 are the main regulators of $H d 3 a$ expression, acting in an independent manner. Ehd1 is a positive regulator of flowering while $\mathrm{Hd} 1$ protein acts as a negative regulator under LD conditions. Thus, the balance between Ehd1 and Hd1 function dictates flowering transition through $\mathrm{Hd} 3 \mathrm{a}$ induction. The adaptation of rice to LD conditions has drawn an alternative regulatory pathway to avoid inhibition exerted by Hd1. One possibility is that an alternative gene, other than $H d 3 a$, orchestrates the phase transition under LD condition. RFT1 has been pointed as the major floral activator under LD conditions

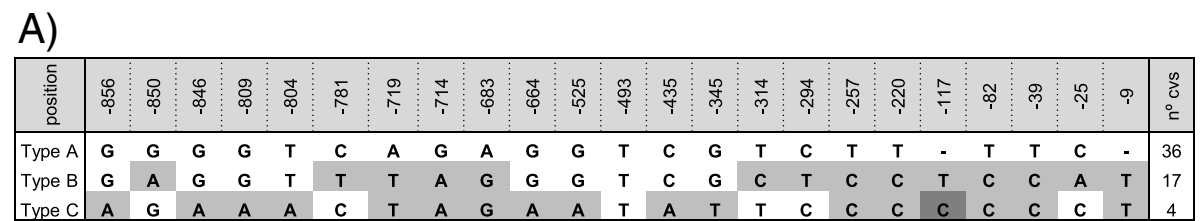

B)

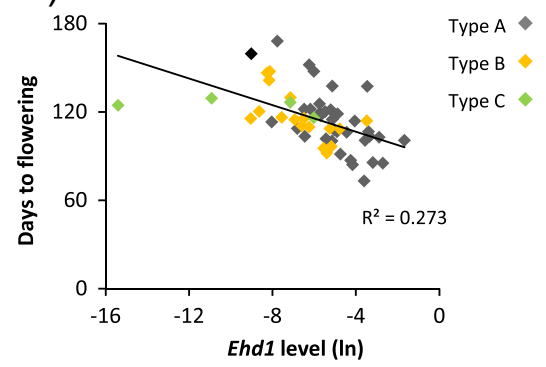

C)

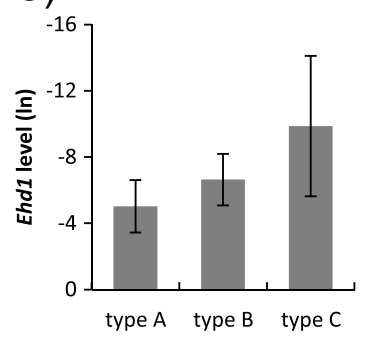

Figure 4 Nucleotide polymorphisms in Ehd1 promoter and its relationship with flowering time. (A) Nucleotide polymorphism in the Ehd1 promoter. The nucleotide sequences in the cultivars were compared with those of Nipponbare (indicated as Type A). Polymorphic nucleotides are indicated in grey. Numbers indicate position from ATG start codon. The number of cultivars containing each type of allele is indicated in the right column. (B) Correlation of flowering time with RNA levels of Ehd 1 in leaves. RNA levels were determined by real-time RT-PCR and shown as natural logarithms. The square of Pearson's product-moment correlation coefficient is indicated $(P<0,05)$. Color dots indicate cultivars carrying different types of Ehd1 alleles. (C) Ehd1 RNA levels in cultivars with type A, B or C Ehd1 promoters. RNA levels represented the mean and are shown as natural logarithms. Error bands represent standard deviation. 


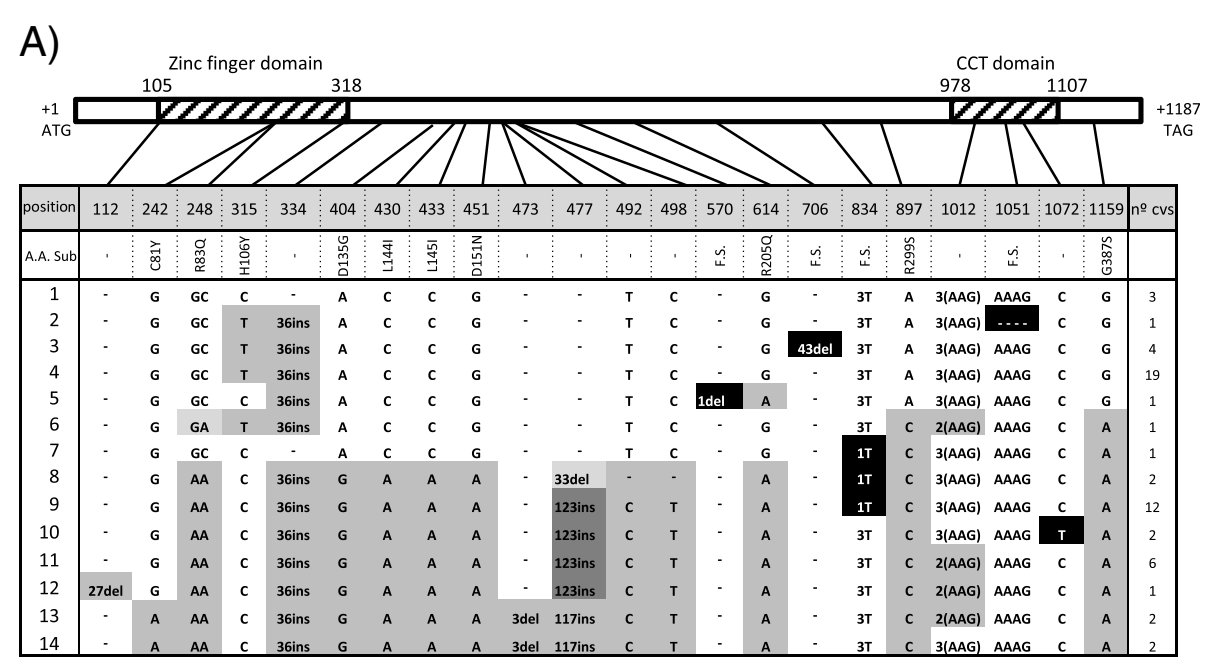

B)
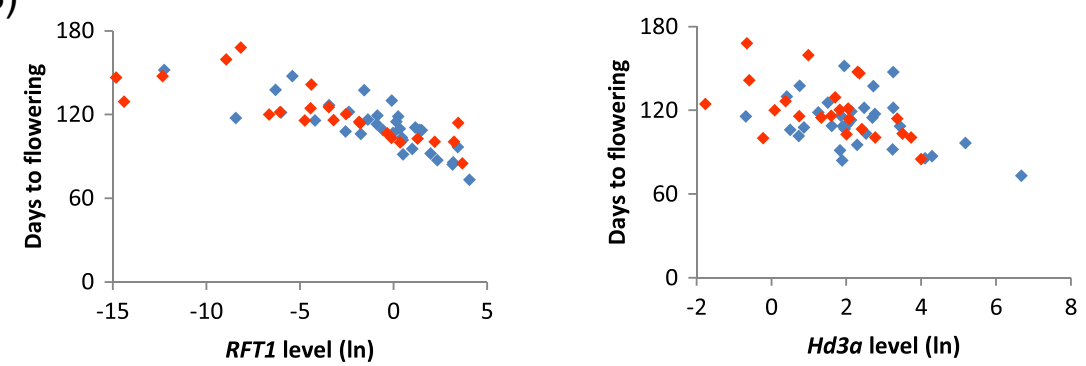

Figure $\mathbf{5}$ Variations in $\mathbf{H d} \mathbf{1}$ and its relationship with $\mathrm{Hd} \mathbf{3 a}$ expression levels. (A) Nucleotide polymorphism in the Hdl coding region. The nucleotide sequences in the cultivars were compared with those of Nipponbare (indicated as Type 1). Polymorphic nucleotides are indicated in black or grey. Amino acid substitutions caused by nucleotide polymorphism are indicated, frame shift is indicated as F.S. Polymorphisms labeled in black caused loss of function alleles. Numbers indicate position from ATG start codon. The number of cultivars containing each type of allele is indicated in the right column. Shaded boxes represent the $\mathrm{Hd} 1$ zinc finger domain and CCT domain. (B) Correlation of flowering time with RFT1 and Hd3a RNA levels of cultivars carrying functional (blue dots) or non-functional Hd1 alleles (red dots). RNA levels were determined by real-time RT-PCR and shown as natural logarithms.

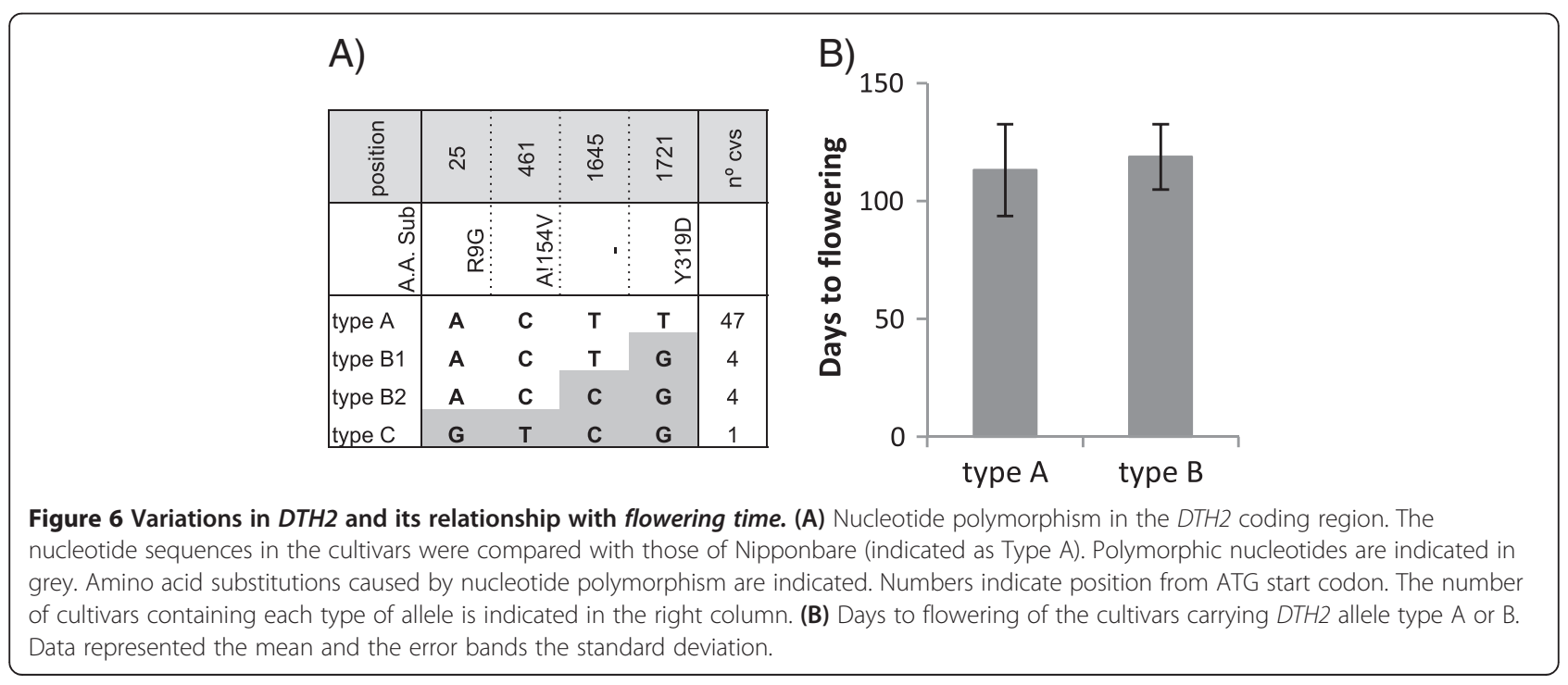


[3]. RFT1 is a member of the FT-like family and shares 91\% of identity with $H d 3 a$, the closest homologue. Suppression of both $H d 3 a$ and RFT1 resulted in no flowering in plants grown under SD conditions, indicating that $H d 3 a$ and RFT1 are the major floral activators in rice under SD conditions [23]. According to current indications, it is believed that $H d 3 a$, but not RFT1, is the pivotal factor promoting floral transition under SD while, RFT1 would play a secondary role in these conditions [23]. In our core collection RFT1 expression showed higher correlation than $H d 3 a$ with days to flowering in plants grown under LD conditions. These data support the hypothesis that RFT1 is the major contributor to flowering under LD conditions in japonica cultivars. Additionally, the possibility that the expression of both RFT1 and $H d 3 a$ affects each other has been investigated, but there is evidence that neither $H d 3 a$ mRNA nor $\mathrm{Hd} 3$ a protein acts directly on the RFT1 promoter [23]. There are common regulation factors recognizing DNA binding sites present in both promoter regions, as ARR1 or CAAT-box containing factors. In that sense, it is known that Ehd1 regulates positively both $H d 3 a$ and RFT1 [3], and it explains that $H d 3 a$ is expressed under LD condition in the absence of $H d 1$ inhibition. It has been described that SDG724 action specifically affects DNA methylation and differential H3K36me2/3 deposition levels at the RFT1 locus but not at the $H d 3 a$ locus [24]. Chromatin modification by SDG724 also induces expression of Ehd1, via MADS50, independently of RFT1 [24]. SDG274 could be a common factor of regulation of both $\mathrm{Hd} 3 \mathrm{a}$ and $R F T 1$, acting in an indirect manner by inducing the expression of Ehd 1 and also in a direct manner on RFT1.

DTH2 modulates $H d 3 a$ and RFT1 expression independently of Ehd1 and $H d 1$. Although it represents a minor-effect quantitative trait locus it has been suggested that DTH2 played an important role in adaptation to specific environments differing in day length [25]. Distribution of DTH2 alleles in cultivated rice in Asia indicated that DTH2 has been a target of artificial selection during domestication under LD conditions. We found a high degree of conservation of DTH2 in our core collection. Most of the cultivars analyzed in this study carried the same allele previously found in japonica cultivars grown in Asia [25], supporting this hypothesis. We also found a second allele in our core collection that contained a polymorphism that caused an amino acid substitution that did not affect the functional domains of the protein and we found no evidence that it may cause any differences in the flowering time.

As expected, RFT1 and $H d 3 a$ are well conserved among the core collection. In the japonica cultivars there is a major allele for each gene. It is thought that RFT1 and $H d 3 a$ were generated by gene duplication followed by divergence [32]. Phylogenetic trees of FTlike genes showed that RFT1 is unique to rice and that it diverged more rapidly than $H d 3 a$ during rice evolution [32]. Gene duplication usually generates functional redundancy and disruption by mutation of the function of one of both genes may lead to a pseudogene. Therefore, two genes with identical functions are unlikely to be stably maintained in the genome, unless both duplicates differ in some aspects of their functions [33,34]. Both RFT1 and Hd3a encode the florigen signal but it appears that they have different roles since their regulation differs according to day length. In that sense, the regulation of flowering time through RFT1 regulation may be part of a mechanism of adaptation of plants to temperate climate conditions. Functional $H d 3$ gene is still present in japonica cultivars and it would explain the capacity to flower under SD conditions of most of the cultivars included in the core collection (unpublished observations).

$H d 1$ is a repressor of flowering under LD conditions through inhibiting $H d 3 a$ expression. Loss-of function alleles of $H d 1$ are common in cultivated rice and it has been suggested that the lack of functionality of $H d 1$ has been a target during domestication to diversify flowering times [28]. We have found 14 different alleles among 57 cultivars, almost half of them are non-functional due to small deletions that caused frame shift or early stop codons. The non-functional alleles were widely distributed across all continents and they were not clearly associated with any location in particular. Similar results were found in previous studies that showed that loss-of function alleles represented more that $50 \%$ of the alleles found in japonica and indica cultivars and 95,5\% in aus cultivars [30]. The high number of $H d 1$ alleles and the presence of non-functional alleles reflect the relaxation of pressure exerted on this gene, probably due to the lower implication of $H d 1$ in flowering under LD conditions and the replacement of its influence by other regulating factors. Furthermore, we have not found statistical differences between the presence of functional alleles and the time of flowering. In previous studies performed mainly with indica rice under SD conditions, cultivars with non-functional $H d 1$ alleles showed significantly later flowering times than those with functional $H d 1$ alleles [27]. However, in our core collection we did not observe any difference in flowering time among japonica cultivars that could be associated with the presence of a functional or non-functional $H d 1$ allele. Additionally, our results indicated that $H d 3 a$ and RFT1 expression levels are not affected by the type of $H d 1$ allele present in each cultivar. This hypothesis appears to be reinforced if RFT1 is considered the main regulator of floral transition. In such model, the influence of $H d 3 a$ and RFT1 in flowering is specific of day length conditions and $H d 3 a$ becomes the major influential factor under SD conditions while RFT1 plays this role under LD conditions. All together indicates that in the cultivars $H d 1$ may not be 
a dominant element determining flowering transition through $H d 3 a$ under LD. The predominant role of RFT1 in flowering induction in plants adapted to LD would represent an advantage by avoiding the inhibitory action of $H d 1$ and, thus relaxing the evolutionary pressure on this gene. In the absence of inhibition by $H d 1$, the activation of RFT1 and Hd3a would be exerted by the action of Ehd1, the flowering signal integrator, although other factors implicated in the regulation of RFT1, as chromatin modification by $S D G 724$, may also play controlling roles.

\section{Conclusions}

Rice was domesticated in a region located in the tropics, from where it extended to other geographical areas reaching latitudes with temperate climate where photoperiod conditions were not favorable for flowering and, thus, for reproduction. To adapt to the new conditions, rice had to overcome or avoid the inhibition action of $H d 1$ under LD length. In these circumstances, two alternatives may be adopted: the emergence of non-functional $H d 1$ alleles that could inhibit flowering under LD conditions and the appearance of an alternative regulatory pathway governed by RFT1. The existence of non-functional $H d 1$ alleles and the lack of correlation of $H d 3 a$ expression levels with the flowering time of the cultivars adapted to LD conditions support the hypothesis that $H d 3 a$ plays a minor role in the regulatory pathway in northern latitudes. Additionally, our data suggested that RFT1 could be the main factor in the regulation of flowering among japonica cultivars adapted to LD conditions. In the absence of inhibition exerted by $H d 1$ through repression of $H d 3 a$, the influence of Ehd1 in the regulation of RFT1 and $H d 3 a$ appeared reinforced. The data also indicated the occurrence of other regulatory factors controlling both master genes.

\section{Methods}

\section{Plant material and growth conditions}

The rice core collection was obtained from different germplasm collections: the International Rice Research Institute (IRRI, Philippines), U.S. National Plant Germplasm System (NPGS, USA), Rice Genome Resource Center (RGRC, Japan) and Instituto Valenciano de Investigaciones Agrarias (IVIA, Spain). Seeds from different cultivars were germinated and grown in pots in greenhouses $\left(39^{\circ} 28^{\prime} \mathrm{N}\right)$ under controlled temperature $\left(25^{\circ} \mathrm{C}\right)$ and $\mathrm{RH}(50 \% \mathrm{RH})$ and natural daylight conditions during summer and were thereafter exposed to more than 13 hour daylight during the whole cycle. Four to 6 independent plants were used to score flowering time. The emergence of the first panicle from the node was recorded and referred as flowering time.

\section{EcoTilling analysis}

To detect allelic polymorphisms present in each cultivar, seeds were germinated and leaf tissue from 15 days seedlings was frozen and DNA extracted, using DNeasy plant mini kit (Qiagen). Specific primers were designed to amplify form 1 to $1,5 \mathrm{~kb}$ fragments of $H d 3 a$, RFT1, Ehd1 or Hd1 (Additional file 1: Table S1). DNA from each cultivar was amplified and the individual PCR products were mixed with IR64 or Nipponbare PCR amplification products in equal amounts and, after denaturing and re-naturing to allow the formation of heteroduplex DNA molecules, they were digested with endonuclease CelI, isolated from celery. Fragments were separated and analyzed by electrophoresis using agarose gels. Cultivars were grouped according to the observed electrophoretic patterns and DNA fragments from five or six cultivars representing each group were sequenced to determine polymorphisms.

\section{RNA isolation and quantitative real-time PCR assays}

Total RNA was isolated with the RNeasy plant mini kit (Qiagen), followed by DNase digestion. The RNA concentration was determined by a fluorometric assay with the kit Quant-iT ${ }^{\mathrm{TM}}$ RiboGreen ${ }^{\odot}$ RNA Assay (Molecular Probes Inc, OR). One-step Real-time PCRs were performed as previously described y on a LightCycler ${ }^{\oplus} 2.0$ (Roche Applied Science, GE Healthcare) using the LightCycler $^{\circ}$ Fast Start DNA MasterPlus Sybr Green I kit (Roche Applied Science, GE Healthcare). One hundred nanograms of total RNA were used for each analysis. Primers used for the analysis are indicated in Supplemental Table 1. The RT-PCR procedure consisted in incubation at $48^{\circ} \mathrm{C}$ for $30 \mathrm{~min}$, followed of 45 cycles at $95^{\circ} \mathrm{C}$ for $2 \mathrm{~s}, 58^{\circ} \mathrm{C}$ for $8 \mathrm{~s}$ and $72^{\circ} \mathrm{C}$ for $13 \mathrm{~s}$. The values presented are the mean of two biological replicates, each with two technical replicates. The error bars indicate the standard deviation from the mean.

\section{Statistical analysis}

Statistical analyses were performed using Statgraphics Plus or Microsoft Office Excel 2007. Correlation between flowering time and gene expression level was examined by Pearson's correlation coefficient test. Comparison between flowering times and $H d 3 a$ expression levels of plants with functional and non-functional Hd1 alleles were determined by the 2-tailed student's test. Differences with $\mathrm{P}<0.01$ were considered to be significant.

\section{Additional file}

Additional file 1: Table S1. Sequences of primers used in EcoTilling and QRT-PCR analysis.

\section{Abbreviations}

bp: Base pair; LD: Long day; In: Natural logarithm; mRNA: Messenger RNA; RT-PCR: Real-time polymerase chain reaction; SD: Short day. 


\section{Competing interests}

The authors declare that they have no competing interests.

\section{Authors' contributions}

CD conceived the project, designed research, and wrote the manuscript. LN performed EcoTilling analysis, RNA isolation and expression analysis, interpretation of data and participated in manuscript preparation. MT designed research, participated in manuscript preparation and revision. All authors read and approved the final manuscript.

\section{Acknowledgements}

This work was supported by the Instituto Nacional de Investigación y Tecnología Agraria y Alimentaria (INIA) (financial support to CD) and by the Ministerio de Ciencia e Innovación of Spain (grant IPT-2011-1244-010000). We thank Cristina Martínez and Matilde Sancho for technical assistance.

Received: 25 June 2013 Accepted: 31 January 2014

Published: 5 February 2014

\section{References}

1. Huang X, Kurata N, Wei X, Wang ZX, Wang A, Zhao Q, Zhao Y, Liu K, Lu H, Li W, Guo Y, Lu Y, Zhou C, Fan D, Weng Q, Zhu C, Huang T, Zhang L, Wang Y, Feng L, Furuumi H, Kubo T, Miyabayashi T, Yuan X, Xu Q, Dong G, Zhan Q, Li C, Fujiyama A, Toyoda A, Lu T, Feng Q, Qian Q, Li J, Han B: A map of rice genome variation reveals the origin of cultivated rice. Nature 2012, 490(7421):497-501.

2. Tamaki S, Matsuo S, Wong HL, Yokoi S, Shimamoto K: Hd3a protein is a mobile flowering signal in rice. Science 2007, 316(5827):1033-1036.

3. Komiya R, Yokoi S, Shimamoto K: A gene network for long-day flowering activates RFT1 encoding a mobile flowering signal in rice. Development 2009, 136(20):3443-3450

4. Hayama R, Yokoi S, Tamaki S, Yano M, Shimamoto K: Adaptation of photoperiodic control pathways produces short-day flowering in rice. Nature 2003, 422(6933):719-722.

5. Robson F, Costa MM, Hepworth SR, Vizir I, Pineiro M, Reeves PH, Putterill J, Coupland G: Functional importance of conserved domains in the flowering-time gene CONSTANS demonstrated by analysis of mutant alleles and transgenic plants. Plant J 2001, 28(6):619-631.

6. Izawa T, Oikawa T, Sugiyama N, Tanisaka T, Yano M, Shimamoto K: Phytochrome mediates the external light signal to repress FT orthologs in photoperiodic flowering of rice. Genes Dev 2002, 16(15):2006-2020.

7. Kojima S, Takahashi Y, Kobayashi Y, Monna L, Sasaki T, Araki T, Yano M: $\mathrm{Hd} 3 \mathrm{a}$, a rice ortholog of the Arabidopsis FT gene, promotes transition to flowering downstream of Hd1 under short-day conditions. Plant Cell Physiol 2002, 43(10):1096-1105.

8. Yano M, Katayose Y, Ashikari M, Yamanouchi U, Monna L, Fuse T, Baba T, Yamamoto K, Umehara Y, Nagamura Y, Sasaki T: Hd1, a major photoperiod sensitivity quantitative trait locus in rice, is closely related to the Arabidopsis flowering time gene CONSTANS. Plant Cell 2000, 12:2473-2484.

9. Ogiso E, Takahashi Y, Sasaki T, Yano M, Izawa T: The role of casein kinase II in flowering time regulation has diversified during evolution. Plant Physiol 2010, 152(2):808-820.

10. Doi K, Izawa T, Fuse T, Yamanouchi U, Kubo T, Shimatani Z, Yano M, Yoshimura A: Ehd1, a B-type response regulator in rice, confers short-day promotion of flowering and controls FT-like gene expression independently of Hd1. Genes Dev 2004, 18(8):926-936.

11. Kim SL, Lee $S$, Kim HJ, Nam HG, An G: OsMADS51 is a short-day flowering promoter that functions upstream of Ehd1, OsMADS14, and Hd3a. Plant Physiol 2007, 145(4):1484-1494.

12. Lee S, Kim J, Han JJ, Han MJ, An G: Functional analyses of the flowering time gene OSMADS50, the putative SUPPRESSOR OF OVEREXPRESSION OF CO 1/AGAMOUS-LIKE 20 (SOC1/AGL20) ortholog in rice. Plant J 2004, 38(5):754-764

13. Ryu CH, Lee S, Cho LH, Kim SL, Lee YS, Choi SC, Jeong HJ, Yi J, Park SJ, Han CD, An G: OsMADS50 and OsMADS56 function antagonistically in regulating long day (LD)-dependent flowering in rice. Plant Cell Environ 2009, 32(10):1412-1427.

14. Wei X, Xu J, Guo H, Jiang L, Chen S, Yu C, Zhou Z, Hu P, Zhai H, Wan J: DTH8 suppresses flowering in rice, influencing plant height and yield potential simultaneously. Plant Physiol 2010, 153(4):1747-1758.

15. Yan WH, Wang $P$, Chen HX, Zhou HJ, Li QP, Wang CR, Ding ZH, Zhang YS, Yu SB, Xing YZ, Zhang QF: A major QTL, Ghd8, plays pleiotropic roles in regulating grain productivity, plant height, and heading date in rice. Mol Plant 2011, 4(2):319-330.

16. Peng LT, Shi ZY, Li L, Shen GZ, Zhang JL: Ectopic expression of OsLFL1 in rice represses Ehd 1 by binding on its promoter. Biochem Biophys Res Commun 2007, 360(1):251-256.

17. Xue W, Xing Y, Weng X, Zhao Y, Tang W, Wang L, Zhou H, Yu S, Xu C, Li X, Zhang Q: Natural variation in Ghd7 is an important regulator of heading date and yield potential in rice. Nat Genet 2008, 40(6):761-767.

18. Matsubara K, Yamanouchi U, Wang ZX, Minobe Y, Izawa T, Yano M: Ehd2, a rice ortholog of the maize INDETERMINATE 1 gene, promotes flowering by up-regulating Ehd1. Plant Physiol 2008, 148(3):1425-1435

19. Park SJ, Kim SL, Lee S, Je BI, Piao HL, Park SH, Kim CM, Ryu CH, Park SH, Xuan $\mathrm{YH}$, Colasanti J, An G, Han CD: Rice Indeterminate 1 (Osld1) is necessary for the expression of Ehd1 (Early heading date 1) regardless of photoperiod. Plant J 2008, 56(6):1018-1029.

20. Gao H, Zheng XM, Fei G, Chen J, Jin M, Ren Y, Wu W, Zhou K, Sheng P, Zhou F, Jiang L, Wang J, Zhang X, Guo X, Wang JL, Cheng Z, Wu C, Wang $H$, Wan JM: Ehd4 encodes a novel and Oryza-genus-specific regulator of photoperiodic flowering in rice. PLoS Genet 2013, 9(2):e1003281.

21. Andres F, Galbraith DW, Talon M, Domingo C: Analysis of PHOTOPERIOD SENSITIVITY5 sheds light on the role of phytochromes in photoperiodic flowering in rice. Plant Physiol 2009, 151(2):681-690.

22. Izawa T: Adaptation of flowering-time by natural and artificial selection in Arabidopsis and rice. J Exp Bot 2007, 58(12):3091-3097.

23. Komiya R, Ikegami A, Tamaki S, Yokoi S, Shimamoto K: Hd3a and RFT1 are essential for flowering in rice. Development 2008, 135(4):767-774.

24. Sun C, Fang J, Zhao T, Xu B, Zhang F, Liu L, Tang J, Zhang G, Deng X, Chen F, Qian Q, Cao X, Chu C: The histone methyltransferase SDG724 mediates H3K36me2/3 deposition at MADS50 and RFT1 and promotes flowering in rice. Plant Cell 2012, 24(8):3235-3247.

25. Wu W, Zheng XM, Lu G, Zhong Z, Gao H, Chen L, Wu C, Wang HJ, Wang Q, Zhou K, Wang JL, Wu F, Zhang X, Guo X, Cheng Z, Lei C, Lin Q, Jiang L, Wang H, Ge S, Wan J: Association of functional nucleotide polymorphisms at DTH2 with the northward expansion of rice cultivation in Asia. Proc Natl Acad Sci U S A 2013, 110(8):2775-2780.

26. Huang CL, Hung CY, Chiang YC, Hwang CC, Hsu TW, Huang CC, Hung KH, Tsai KC, Wang KH, Osada N, Schaal BA, Chiang TY: Footprints of natural and artificial selection for photoperiod pathway genes in Oryza. Plant J 2012, 70(5):769-782

27. Takahashi Y, Teshima KM, Yokoi S, Innan H, Shimamoto K: Variations in Hd1 proteins, Hd3a promoters, and Ehd1 expression levels contribute to diversity of flowering time in cultivated rice. Proc Natl Acad Sci USA 2009, 106(11):4555-4560.

28. Takahashi Y, Shimamoto K: Heading date $1(\mathrm{Hd} 1)$, an ortholog of Arabidopsis CONSTANS, is a possible target of human selection during domestication to diversify flowering times of cultivated rice. Genes Genet Syst 2011, 86(3):175-182.

29. Tsuji H, Taoka K, Shimamoto K: Regulation of flowering in rice: two florigen genes, a complex gene network, and natural variation. Curr Opin Plant Biol 2011, 14(1):45-52

30. Fujino K, Wu J, Sekiguchi H, Ito T, Izawa T, Matsumoto T: Multiple introgression events surrounding the $\mathrm{Hd} 1$ flowering-time gene in cultivated rice, Oryza sativa L. Mol Genet Genomics 2010, 284(2):137-146.

31. Courtois B, Frouin J, Greco R, Bruschi G, Droc G, Hamelin C, Ruiz M, Clément G, Evrard J, van Coppenole S, Katsantonis D, Oliveira M, Negrão S, Matos C, Cavigiolo S, Lupotto E, Piffanelli P, Ahmadi N: Genetic diversity and population structure in a European collection of rice. Crop Sci 2012, 52:1663-1675

32. Hagiwara WE, Uwatoko N, Sasaki A, Matsubara K, Nagano H, Onishi K, Sano $Y$ : Diversification in flowering time due to tandem FT-like gene duplication, generating novel Mendelian factors in wild and cultivated rice. Mol Ecol 2009, 18(7):1537-1549.

33. Force A, Lynch M, Pickett FB, Amores A, Yan YL, Postlethwait J: Preservation of duplicate genes by complementary, degenerative mutations. Genetics 1999, 151(4):1531-1545.

34. Lynch M, O'Hely M, Walsh B, Force A: The probability of preservation of a newly arisen gene duplicate. Genetics 2001, 159(4):1789-1804.

doi:10.1186/1471-2164-15-101

Cite this article as: Naranjo et al.: Diversity of floral regulatory genes of japonica rice cultivated at northern latitudes. BMC Genomics 2014 15:101. 